\title{
CENTRALISERS ON RINGS AND ALGEBRAS
}

\author{
Joso VUKMan and IRENA KOSI-UlBL
}

\begin{abstract}
In this paper we investigate identities related to centralisers in rings and algebras. We prove, for example, the following result. Let $A$ be a semisimple $H^{*}$-algebra and let $T: A \rightarrow A$ be an additive mapping satisfying the relation $T\left(x^{m+n+1}\right)$ $=x^{m} T(x) x^{n}$ for all $x \in A$ and some integers $m \geqslant 1, n \geqslant 1$. In this case $T$ is a left and a right centraliser.
\end{abstract}

Throughout, $R$ will represent an associative ring with centre $Z(R)$. Given an integer $n \geqslant 2$, a ring $R$ is said to be $n$-torsion free, if for $x \in R, n x=0$ implies $x=0$. As usual the commutator $x y-y x$ will be denoted by $[x, y]$. Recall that a ring $R$ is prime if for $a, b \in R, a R b=(0)$ imples that either $a=0$ or $b=0$, and is semiprime in case $a R a=(0)$ imples $a=0$. An additive mapping $T: R \rightarrow R$ is called a left centraliser in case $T(x y)=T(x) y$ holds for all $x, y \in R$. The concept appears naturally in $C^{*}$-algebras. In ring theory it is more common to work with module homomorphisms. Ring theorists would write that $T: R_{R} \rightarrow R_{R}$ is a homomorphism of a ring module $R$ into itself. For a semiprime ring $R$ all such homomorphisms are of the form $T(x)=q x$ where $q$ is an element of the Martindale right ring to quotients $Q_{r}$ (see Chapter 2 by Beidar and Martindale). In case $R$ has the identity element $T: R \rightarrow R$ is a left centraliser if and only if $T$ is of the form $T(x)=a x$ for some $a \in R$. An additive mapping $T: R \rightarrow R$ is called a left Jordan centraliser in case $T\left(x^{2}\right)=T(x) x$ holds for all $x \in R$. The definition of right centralizer and right Jordan centralizer should be self-explanatory. In case $T: R \rightarrow R$ is a left and right centraliser, where $R$ is a semiprime ring with extended centroid $C$, then there exists an element $\lambda \in C$ such that $T(x)=\lambda x$ for all $x \in R$ (see [2, Theorem 2.3.2]).

Zalar [12] has proved that any left (right) Jordan centraliser on a 2-torsion free semiprime ring is a left (right) centraliser. Molnár [7] has proved that in case we have an additive mapping $T: A \rightarrow A$, where $A$ is a semisimple $H^{*}$-algebra, satisfying the relation $T\left(x^{3}\right)=T(x) x^{2}$ (respecitvely $T\left(x^{3}\right)=x^{2} T(x)$ ) for all $x \in A$, then $T$ is a left (right) centraliser. Let us recall that a semisimple $H^{*}$-algebra is a semisimple Banach * -algebra whose norm is a Hilbert space norm such that $\left(x, y z^{*}\right)=(x z, y)=\left(z, x^{*} y\right)$

Received 20th October, 2004

This research has been supported by the Research Council of Slovenia.

Copyright Clearance Centre, Inc. Serial-fee code: 0004-9727/05 \$A2.00+0.00. 
is fulfilled for all $x, y, z \in A$ (see [1]). The result of Benkovič and Eremita [3] states that in case we have a prime ring $R$ and an additive mapping $T: R \rightarrow R$ satisfying the relation $T\left(x^{n}\right)=T(x) x^{n-1}$ for all $x \in R$, where $n \geqslant 2$ is a fixed integer, then $T$ is a left centraliser in case $\operatorname{char}(R)=0$ or $\operatorname{char}(R) \geqslant n$. Some results concerning centalisers on semiprime rings can be found in $[3,6]$ and $[8,9,10,11]$. Let $X$ be a real or complex Banach space and let $L(X)$ and $F(X)$ denote the algebra of all bounded linear operators on $X$ and the ideal of all finite rank operators in $L(X)$, respectively. An algebra $A(X) \subset L(X)$ is said to be standard in case $F(X) \subset A(X)$. Let us point out that any standard algebra is prime, which is a consequence of Hahn-Banach theorem. We denote by $X^{*}$ the dual space of a Banach space $X$ and by $I$ the identity operator on $X$.

It is our aim in this paper to prove the following result.

TheOREM 1. Let $A$ be a semisimple $H^{*}$-algebra and let $T: A \rightarrow A$ be an additive mapping satisfying the relation

$$
T\left(x^{m+n+1}\right)=x^{m} T(x) x^{n}
$$

for all $x \in A$ and some integers $m \geqslant 1, n \geqslant 1$. In this case $T$ is a left and a right centraliser.

For the proof of the theorem above we need the result below which is of independent interest.

THEOREM 2. Let $X$ be a Banach space over a real or complex field $F$ and let $A(X) \subset L(X)$ be a standard operator algebra. Suppose there exists an additive mapping $T: A(X) \rightarrow L(X)$ satisfying the relation

$$
T\left(A^{m+n+1}\right)=A^{m} T(A) A^{n}
$$

for all $A \in A(X)$ and some integers $m \geqslant 1, n \geqslant 1$. In this case $T$ is of the form $T(A)=\lambda A$ for some $\lambda \in F$.

In the proof of Theorem 2 we shall use some ideas similar to those used in [7] and the following purely algebraic results proved by Brešar [4] and Zalar [12].

Theorem A. ([4, Theorem 2].) Let $R$ be a 2-torsion free prime ring. Suppose there exists an additive mapping $F: R \rightarrow R$ satisfying the relation $[[F(x), x], x]=0$ for all $x \in R$. In this case $[F(x), x]=0$ holds for all $x \in R$.

Theorem B. ([12, Proposition 1.4].) Let $T$ be a 2-torsion free semiprime ring and let $T: R \rightarrow R$ be a left (right) Jordan centraliser. In this case $T$ is a left (right) centraliser.

PROOF OF THEOREM 2: We have the relation

$$
T\left(A^{m+n+1}\right)=A^{m} T(A) A^{n} .
$$


Let us first consider the restriction of $T$ on $F(X)$. Let $A$ be from $F(X)$ and let $P \in F(X)$ be a projection with $A P=P A=A$. From the above relation one obtains $T(P)=P T(P) P$, which gives

$$
T(P) P=P T(P)=P T(P) P \text {. }
$$

Putting $A+P$ for $A$ in the relation (1), we obtain

$$
\begin{aligned}
& \sum_{i=0}^{m+n+1}\left(\begin{array}{c}
m+n+1 \\
i
\end{array}\right) T(\left.A^{m+n+1-i} P^{i}\right) \\
&=\left(\sum_{i=0}^{m}\left(\begin{array}{c}
m \\
i
\end{array}\right) A^{m-i} P^{i}\right)(T(A)+B)\left(\sum_{i=0}^{n}\left(\begin{array}{c}
n \\
i
\end{array}\right) A^{n-i} P^{i}\right),
\end{aligned}
$$

where $B$ stands for $T(P)$. Using (1) and rearranging the equation (3) in the sense of collecting together terms involving an equal number of factors of $P$ we obtain:

$$
\sum_{i=1}^{m+n} f_{i}(A, P)=0
$$

where $f_{i}(A, P)$ stands for the expression of terms involving $i$ factors of $P$. Replacing $A$ by $A+2 P, A+3 P, \ldots, A+(m+n) P$ in turn in the equation (1), and expressing the resulting system of $m+n$ homogeneous equations in the variables $f_{i}(A, P)$, $i=1,2, \ldots, m+n$, we see that the coefficient matrix of the system is a van der Monde matrix

$$
\left[\begin{array}{cccc}
1 & 1 & \cdots & 1 \\
2 & 2^{2} & \cdots & 2^{m+n} \\
\vdots & \vdots & \vdots & \vdots \\
m+n & (m+n)^{2} & \cdots & (m+n)^{m+n}
\end{array}\right]
$$

Since the detminant of the matrix is different from zero, it follows that the system has only a trivial solution.

In particular

$$
\begin{aligned}
& f_{m+n-1}(A, P) \\
& =\left(\begin{array}{l}
m+n+1 \\
m+n-1
\end{array}\right) T\left(A^{2}\right)-\left(\begin{array}{c}
m \\
m-2
\end{array}\right)\left(\begin{array}{l}
n \\
n
\end{array}\right) A^{2} B-\left(\begin{array}{c}
m \\
m
\end{array}\right)\left(\begin{array}{c}
n \\
n-2
\end{array}\right) B A^{2} \\
& \quad-\left(\begin{array}{c}
m \\
m-1
\end{array}\right)\left(\begin{array}{l}
n \\
n
\end{array}\right) A T(A) P-\left(\begin{array}{l}
m \\
m
\end{array}\right)\left(\begin{array}{c}
n \\
n-1
\end{array}\right) P T(A) A-\left(\begin{array}{c}
m \\
m-1
\end{array}\right)\left(\begin{array}{c}
m \\
n-1
\end{array}\right) A B A \\
& =0,
\end{aligned}
$$


and

$$
\begin{aligned}
f_{m+n} & (A, P) \\
= & \left(\begin{array}{c}
m+n+1 \\
m+n
\end{array}\right) T(A)-\left(\begin{array}{c}
m \\
m-1
\end{array}\right)\left(\begin{array}{l}
n \\
n
\end{array}\right) A B-\left(\begin{array}{c}
m \\
m
\end{array}\right)\left(\begin{array}{c}
n \\
n-1
\end{array}\right) B A-\left(\begin{array}{c}
m \\
m
\end{array}\right)\left(\begin{array}{l}
n \\
n
\end{array}\right) P T(A) P \\
= & 0 .
\end{aligned}
$$

The above equations reduce to

(5) $(m+n+1)(m+n) T\left(A^{2}\right)=m(m-1) A^{2} B+n(n-1) B A^{2}+2 m n A B A$

$$
+2 m A T(A) P+2 n P T(A) A,
$$

and

$$
(m+n+1) T(A)=m A B+n B A+P T(A) P .
$$

Right multiplications of the relation (6) by $P$ gives

$$
(m+n+1) T(A) P=m A B+n B A+P T(A) P .
$$

Similarly one obtains

$$
(m+n+1) P T(A)=m A B+n B A+P T(A) P .
$$

Combining (7) with (8) gives

$$
T(A) P=P T(A),
$$

which reduces the relations (5) to

(9) $(m+n+1)(m+n) T\left(A^{2}\right)=m(m-1) A^{2} B+n(n-1) B A^{2}+2 m n A B A$

$$
+2 m A T(A)+2 n T(A) A,
$$

and the relation (7) to

$$
(m+n) T(A) P=m A B+n B A .
$$

Combining (10) with (6) gives

$$
T(A)=T(A) P .
$$

From the above relation one can conclude that $T$ maps $F(X)$ into itself. Further from (11), (10) reduces to

$$
(m+n) T(A)=m A B+n B A .
$$


From this we can conclude that $T$ is linear on $F(X)$. Further apply (12) we obtain

$$
\begin{aligned}
2 m n A B A & =n(m A B) A+m A(n B A) \\
& =n((m+n) T(A)-n B A) A+m A((m+n) T(A)-m A B) \\
& =(m+n)(n T(A) A+m A T(A))-n^{2} B A^{2}-m^{2} A^{2} B .
\end{aligned}
$$

We have therefore

$$
2 m n A B A=(m+n)(n T(A) A+m A T(A))-n^{2} B A^{2}-m^{2} A^{2} B
$$

Applying (12) and the relation above to (9) we obtain

$$
(m+n) T\left(A^{2}\right)=n T(A) A+m A T(A),
$$

and multiplying by $(m+n)$ we obtain

$$
(m+n)^{2} T\left(A^{2}\right)=n(m+n) T(A) A+m A(m+n) T(A) .
$$

Applying the above relation on both sides of (12) we obtain

$$
(m+n)\left(m A^{2} B+n B A^{2}\right)=n(m A B+n B A) A+m A(m A B+n B A),
$$

which reduces to

$$
[[A, B], A]=0 \text {. }
$$

Relation (12) gives $(m+n)[T(A), A]=m A[B, A]+n[B, A] A$. By the above relation one can replace $A[B, A]$ by $[B, A] A$ which gives

$$
[T(A), A]=[B, A] A \text {. }
$$

Then applying (14) we obtain $[[T(A), A], A]=[[B, A] A, A]=[[B, A], A] A=0$. Thus we have

$$
[[T(A), A], A]=0
$$

for any $A \in F(X)$. We have therefore an additive mapping $T$ which maps $F(X)$ into itself satisfying the relation above for any $A \in F(X)$. Since $F(X)$ is prime all the assumptions of Theorem $\mathrm{A}$ are fulfilled which means that

$$
[T(A), A]=0
$$

holds for any $A \in F(X)$. Applying this in (13), one obtains that $T\left(A^{2}\right)=T(A) A$ and $T\left(A^{2}\right)=A T(A)$ holds for all $A \in F(X)$. In other words, $T$ is a left and a right 
Jordan centraliser on $F(X)$. By Theorem B it follows that $T$ is a left and also a right centraliser of $F(X)$.

We intend to prove that there exists $C \in L(X)$, such that

$$
T(A)=C A, \text { for all } A \in F(X) .
$$

For any fixed $x \in X$ and $f \in X^{*}$ we denote by $x \otimes f$ an operator from $F(X)$ defined by $(x \otimes f) y=f(y) x$, for all $y \in X$. For any $A \in L(X)$ we have $A(x \otimes f)=((A x) \otimes f)$. Let us choose $f$ and $y$ such that $f(y)=1$ and define $C x=T(x \otimes f) y$. Obviously, $C$ is linear. Using the fact that $T$ is a left centraliser on $F(X)$ we obtain

$$
(C A) x=C(A x)=T((A x) \otimes f) y=T(A(x \otimes f)) y=T(A)(x \otimes f) y=T(A) x, x \in X .
$$

We have therefore $T(A)=C A$, for any $A \in F(X)$. Since $T$ a right centraliser on $F(X)$ we obtain $C(A P)=T(A P)=A T(P)=A C P$, where $A \in F(X)$ and $P$ is an arbitrary one-dimensional projection. We have therfore $[A, C] P=0$. Since $P$ is arbitrary one-dimensional projection it follows that $[A, C]=0$, for any $A \in F(X)$. Using the closed graph theorem one can easily prove that $C$ is continuous. Since $C$ commutes with all operators from $F(X)$ one can conclude that $C x=\lambda x$ holds for any $x \in X$ and some $\lambda \in F$, which together with the relation (15) gives that $T$ is of the form

$$
T(A)=\lambda A
$$

any $A \in F(X)$ and some $\lambda \in F$.

It remains to prove that the above relation holds for any $A \in A(X)$ as well. Let us introduce $T_{1}: A(X) \rightarrow L(X)$ by $T_{1}(A)=\lambda A$ and consider $T_{0}=T-T_{1}$. The mapping $T_{0}$ is, obviously additive and satisfies the relation (1). Besides, $T_{0}$ vanishes on $F(X)$. It is our aim to prove that $T_{0}$ vanishes on $A(X)$ as well. Let $A \in A(X)$, let $P$ be a one-dimensional projection and let $S=A+P A P-(A P+P A)$. Note that $S$ can be written in the form $S=(I-P) A(I-P)$, where $I$ denotes the identity operator on $X$. Since, obviously, $S-A \in F(X)$, we have $T_{0}(S)=T_{0}(A)$. Besides, $S P=P S=0$. We have therefore the relation

$$
T_{0}\left(A^{m+n+1}\right)=A^{m} T_{0}(A) A^{n},
$$

for all $A \in A(X)$. Applying the above relation we obtain

$$
\begin{aligned}
S^{m} T_{0}(S) S^{n} & =T_{0}\left(S^{m+n+1}\right)=T_{0}\left(S^{m+n+1}+P\right)=T_{0}\left((S+P)^{m+m+1}\right) \\
& =(S+P)^{m} T_{0}\left(S+\left(S^{m}+P\right)\right) T_{0}(S)\left(S^{n}+P\right) \\
& =S^{m} T_{0}(S) S^{n}+P T_{0}(S) S^{n}+S^{m} T_{0}(S) P+P T_{0}(S) P .
\end{aligned}
$$


We have therefore

$$
P T_{0}(S) S^{n}+S^{m} T_{0}(A) P+P T_{0}(A) P=0 .
$$

Multiplying the above relation from both sides by $P$ we obtain

$$
P T_{0}(A) P=0
$$

which reduces (18) to

$$
P T_{0}(A) S^{n}+S^{m} T_{0}(A) P=0 .
$$

Right multiplication by $P$ then gives

$$
S^{m} T_{0}(A) P=0 .
$$

We intend to prove that

$$
S^{m-1} T_{0}(A) P=0 .
$$

Putting $A+B$ for $A$, where $B \in F(X)$, in (21) and using the fact that $T_{0}$ vanishes on $F(X)$, we obtain

$$
\left(S_{1} S^{m-1}+S S_{1} S^{m-2}+\cdots+S^{m-1} S_{1}\right) T_{0}(A) P=0,
$$

where $S_{1}$ stands for $(I-P) B(I-P)$ (see [5]). The substitution $T(A) P B$ for $B$ in the above relation gives because of (19)

$$
\left(T_{0}(A) P B S^{m-1}+S T_{0}(A) P B S^{m-2}+\cdots+S^{m-1} T(A) P B\right) T_{0}(A) P=0 .
$$

Multiplying from the left side by $S^{m-1}$ and applying (21) we obtain

$$
\left(S^{m-1} T_{0}(A) P\right) B\left(S^{m-1} T_{0}(A) P\right)=0,
$$

for all $B \in F(X)$. Then it follows $S^{m-1} T_{0}(A) P=0$ by the primeness of $F(X)$, which proves (22).

Now, (21) implies (22), one can conclude by induction that $S T_{0}(A) P=0$, which gives

$$
A T_{0}(A) P-P A T_{0}(A) P=0,
$$

because of (19). Then putting $A+B$ for $A$, where $B \in F(X)$, we obtain $0=(A+B) T_{0}(A) P-P(A+B) T_{0}(A) P=B T_{0}(A) P-P B T_{0}(A) P$. We have therefore proved that

$$
B T_{0}(A) P-P B T_{0}(A) P=0
$$


holds for all $A \in A(X)$ and all $B \in F(X)$. The substitution $T_{0}(A) P B$ for $B$ in the above relation gives, because of $(19),\left(T_{0}(A) P\right) B\left(T_{0}(A) P\right)=0$, for all $B \in F(X)$. Thus it follows $T_{0}(A) P=0$ by the primeness of $F(X)$. Since $P$ is an arbitrary onedimensional projection, one can conclude that $T_{0}(A)=0$, for any $A \in A(X)$, which completes the proof of the theorem.

ProOF OF THEOREM 1: The proof goes through using the same arguments as in the proof of the Theorem of [7], with the exception that one has to use Theorem 2 instead of the Lemma in [7].

In the proof of Theorem 2 (the relation (13)) we met an additive mapping $T$ : $F(X) \rightarrow F(X)$ satisfying the relation

$$
(m+n) T\left(A^{2}\right)=m A T(A)+n T(A) A
$$

for all $A \in F(X)$. In the case $m=n$ this reduces to $2 T\left(A^{2}\right)=T(A) A+A T(A)$. Vukman [7] has proved that when we have an additive mapping $T: R \rightarrow R$, where $R$ is an arbitrary 2-torsion free semiprime ring, satisfying the relation $2 T\left(x^{2}\right)=T(x) x$ $+x T(x)$ for all $x \in R$, then $T$ is a left and right centraliser. These observations lead to the following conjecture.

Conjecture 1. Let $m$ and $n, m \neq-n$ be some nonzero integers and let $R$ be a semiprime ring with suitable torsion restrictions. Suppose there exists an additive mapping $T: R \rightarrow R$ satisfying the relation

$$
(m+n) T\left(x^{2}\right)=m x T(x)+n T(x) x
$$

for all $x \in R$. In this case $T$ is a left and right centraliser.

Our last result is related to conjecture above.

THEOREM 3. Let $m$ and $n, m \neq-n$, be some nonzero integers and let $R$ be a $|m n|$ and $|m+n|$-torsion free semiprime ring. Suppose there exists and additive mapping $T: R \rightarrow R$ satisfying the relation

$$
(m+n) T(x y)=m x T(y)+n T(x) y ;
$$

for all pairs $x, y \in R$. In this case $T$ is a left and a right centraliser.

Proof: We have the relation

$$
(m+n) T(x y)=m x T(y)+n T(x) y,
$$

for all pairs $x, y \in R$. We compute the expression $(m+n)^{2} T(x y x)$ in two ways. First applying the relation above

$$
\begin{aligned}
(m+n)^{2} T(x(y x)) & =m(m+n) x T(y x)+n(m+n) T(x) y x \\
& =m x(m y T(x)+n T(y) x)+n(m+n) T(x) y x, \quad x, y \in R .
\end{aligned}
$$


Thus we have

$$
(m+n)^{2} T(x y x)=m^{2} x y T(x)+m n x T(y) x+m n T(x) y x+n^{2} T(x) y x,
$$

for $x, y \in R$. On the other hand using (23)

$$
\begin{aligned}
(m+n)^{2} T((x y) x) & =m(m+n) x y T(x)+n(m+n) T(x y) x \\
& =m(m+n) x y T(x)+n(m x T(y)+n T(x) y) x, \quad x, y \in R .
\end{aligned}
$$

Thus we have

$$
(m+n)^{2} T(x y x)=m^{2} x y T(x)+m n x y T(x)+m n x T(y) x+n^{2} T(x) y x ; x, y \in R .
$$

Subtracting the relation (25) from (24) we obtain $m n(T(x) y x=x y T(x))=0$, for all pairs $x, y \in R$, which reduces to

$$
T(x) y x-x y T(x)=0, \quad x, y \in R
$$

since we have assumed that $R$ is $|m n|$-torsion free. Putting in the above relation first $y x$ for $y$ then multiplying from the right side by $x$ and subtracting the relations so obtained one from another we obtain $x y[T(x), x]=0$, for all pairs $x, y \in R$. From this one obtains easily $[T(x), x] y[T(x), x]=0$, for all pairs $x, y \in R$. Hence it follows

$$
[T(x), x]=0, x \in R
$$

by the semiprimeness of $R$. The substitution $y=x$ in (23) gives

$$
(m+n) T\left(x^{2}\right)=m x T(x)+n T(x) x, x \in R .
$$

By (26) one can then replace $x T(x)$ by $T(x) x$ which gives $(m+n) T\left(x^{2}\right)$ $=(m+n) T(x) x$ for all $x \in R$. Since we have assumed that $R$ is $|m+n|$-torsion free, it follows that $T\left(x^{2}\right)=T(x) x$ holds for all $x \in R$. Of course, we also have $T\left(x^{2}\right)=x T(x)$, for all $x \in R$. In other words, $T$ is a left and right Jordan centraliser. By Theorem B $T$ is a left and a right centraliser. The proof of the theorem is complete.

We conclude with the following conjecture.

CONJECTURE 2. Let $R$ be a semiprime ring with suitable torsion restrictions and let $T: R \rightarrow R$ be an additive mapping satisfying the relation

$$
T\left(x^{m+n+1}\right)=x^{m} T(x) x^{n}
$$

for all $x \in R$ and some integers $m \geqslant 1, n \geqslant 1$. In this case $T$ is a left and right centraliser. 


\section{REFERENCES}

[1] W. Ambrose, 'Structure theorems for a special class of Banach algebras', Trans. Amer. Math. Soc. 57 (1945), 364-386.

[2] K.I. Beidar, W.S. Martindale III and A.V. Mikhalev, Rings with generalized indentities (Marcel Dekker Inc., New York, 1996).

[3] D. Benkovič and D. Eremita, 'Characterizing left centralizers by their action on a polynomial', Publ. Math. Debrecen (to appear).

[4] M. Brešar, 'On a generalization of the notion of centralizing mappings', Proc. Amer. Math. Soc 114 (1992), 641-649.

[5] L.O. Chung and J. Luh, 'Semiprime rings with nilpotent elements', Canad. Math. Bull. 24 (1981), 415-421.

[6] I. Kosi-Ulbl, 'A remark on centralizers in semiprime rings', Glas. Mat. Ser. III 39 (2004), 21-26.

[7] L. Molnár, 'On centralizers of an $H^{*}$-algebra', Publ. Math. Debrecen 46 (1995), 89-95.

[8] J. Vukman, 'An identity related to centralizers in semiprime rings', Comment. Math. Univ. Carolin. 40 (1999), 447-456.

[9] J. Vukman, 'Centralizers of semiprime rings', Comment. Math. Univ. Carolin. 42 (2001), 237-245.

[10] J. Vukman and I. Kosi Ulbl, 'On centralizers of semiprime rings', Aequationes Math. 66 (2003), 277-283.

[11] J. Vukman and I. Kosi-Ulbl, 'An equation realted to dentralizers in semiprime rings', Glas. Mat. Ser. III 38 (2003), 253-261.

[12] B. Zalar, 'On centralizers of semiprime rings', Comment. Math. Univ. Carolin. 32 (1991), 609-614.

\footnotetext{
Department of Mathematics

University of Maribor

PEF, Koroška 160

2000 Maribor

Slovenia

e-mail: joso.vukman@uni-mb.si irena.kosi@uni-mb.si
} 